27.9.-29.9. 1999 Jahrestagung der dvs-Kommission Frauenforschung in der Sportwissenschaft: „Sportwissenschaft im Diskurs des Postfeminismus"

Tagungsort: Heidelberg

Veranstalter: dvs-Kommission Frauenforschung in der Sportwissenschaft

Kontakt: Prof. Dr. Claudia Kugelmann, Universität ErlangenNürnberg, Institut für Sportwissenschaft, Regensburger Str. 160, 90478 Nürnberg, Tel.: (0911) 5302-568, Fax: (09 11) 5302578, E-Mail: claudia.kugelmann@ewf.uni-erlangen.de

\title{
Sportwissenschaft aktuell
}

\section{Deutscher Sportbund Hermann-Altrock-Stipendium 1999}

Das Stipendium soll Promovierenden in der Sportwissenschaft Beginn oder Fertigstellung einer thematisch wichtigen Dissertation ermöglichen. Es hat eine Laufzeit von einem Jahr, die monatliche Unterstützung beträgt DM 1000,--

Vorschläge für die Verleihung sind bis zum 15. August 1999 an den Deutschen Sportbund, Abteilung Bildung und Wissenschaft, Otto-Fleck-Schneise 12, 60528 Frankfurt/M., zu richten.

Einzureichen sind zusammen mit Referenzen von zwei Hochschullehrern/Hochschullehrerinnen und einem handschriftlichen Lebenslauf des Bewerbers/der Bewerberin (mit Lichtbild), in dem dieser/diese u. a. die beabsichtigte Verwendung des Stipendiums darlegt: Zeugnis über den Abschluß des sportwissenschaftlichen Studiums; Angebot über Thema, Aufbau und Stand des Promotionsvorhabens, Bericht über die Mitarbeit im Studium, insbesondere in Seminaren und an Forschungsvorhaben, Bericht über ehrenamtliche, neben- oder hauptberufliche Tätigkeiten im organisierten Sport.

Die Entscheidung über die Bewerbungen trifft der Deutsche Sportbund. Bei gleicher Qualifikation wird Bewerberinnen der Vorzug gegeben. 